\title{
Aging Monitoring Method of Bond Wires Based on Phase-Frequency Characteristics of Differential Mode Conducted Interference Signal for IGBT Module
}

\author{
Chu, Chengpeng; Dong, Chao; Hu, Jing; Du, Mingxing; Ouyang, Ziwei
}

Published in:

IEEE Transactions on Device and Materials Reliability

Link to article, DOI:

10.1109/TDMR.2021.3125344

Publication date:

2021

Document Version

Peer reviewed version

Link back to DTU Orbit

Citation (APA):

Chu, C., Dong, C., Hu, J., Du, M., \& Ouyang, Z. (2021). Aging Monitoring Method of Bond Wires Based on

Phase-Frequency Characteristics of Differential Mode Conducted Interference Signal for IGBT Module. IEEE

Transactions on Device and Materials Reliability, 21(4), 639-646. https://doi.org/10.1109/TDMR.2021.3125344

\section{General rights}

Copyright and moral rights for the publications made accessible in the public portal are retained by the authors and/or other copyright owners and it is a condition of accessing publications that users recognise and abide by the legal requirements associated with these rights.

- Users may download and print one copy of any publication from the public portal for the purpose of private study or research.

- You may not further distribute the material or use it for any profit-making activity or commercial gain

- You may freely distribute the URL identifying the publication in the public portal 


\title{
Aging Monitoring Method of Bond Wires Based on Phase-Frequency Characteristics of Differential Mode Conducted Interference Signal for IGBT Module
}

\author{
Chengpeng Chu, Chao Dong, Jing Hu, Mingxing Du and Ziwei Ouyang, Senior Member, IEEE
}

\begin{abstract}
This paper presents an on-line monitoring method of IGBT module bond wires aging based on the phase-frequency characteristics (PFCs) of differential mode (DM) interference signal. Firstly, the collector current variation during IGBT chip switching is analyzed, and the relationship between the aging degree of bond wires and the PFCs of DM interference signal is studied; Secondly, a phase angle characteristic measurement system of DM interference signal is built and used to online monitor the aging of bond wires in IGBT module; Finally, taking the buck converter composed of IGBT module as the equipment under tested, the interference signal is extracted by the RF current probe and processed by the low-pass filter and signal amplifier, then the phase angle characteristics are measured by the oscilloscope board to realize the on-line monitoring of the bond wires aging of IGBT module. The experimental results show that compared with buck converter with healthy IGBT module, the phase angle of DM interference signal in Buck Converter with aging IGBT module is reduced. The proposed method does not need expensive equipment such as spectrum analyzer, and only needs to monitor the aging state of bond wires in IGBT module online through oscilloscope board. Besides, the research method in this paper provides an approximate linear monitoring method for bonding wire lift-off.
\end{abstract}

Index Terms - On-line monitoring, bond wires, differential mode conducted interference, insulate gate bipolar transistor, phase-frequency characteristics

\section{INTRODUCTION}

$I^{-1+4}$ GBT is one of the core components of power electronic system to realize power conversion and control[1]. Due to the turn-on and turn-off of IGBT module and the change of external operation environment, IGBT module bears unbalanced thermal stress for a long time. During the operation, the thermal fatigue of the package structure is easy to reduce, thus reducing its reliability and aging gradually [2] [3]. In the working process of IGBT module, due to the different coefficient of thermal

This work was supported in part by and Tianjin Municipal Science and Technology Project (No.20YDTPJC00510) and Scientific Research Program of Tianjin Education Commission (No.2018KJ162). (Corresponding Author: Mingxing Du.)

Chengpeng Chu, Chao Dong, Mingxing Du, Ziwei Ouyang are with Tianjin Key Laboratory of Control Theory \& Applications in Complicated System, Tianjin University of Technology, Tianjin, China. (e- expansion (CTE) between the chip and the bond wires, different shear stress is generated between them, which leads to the bond wires lift off [4]. The aging of IGBT module bond wires not only effects the performance of power converter, but also may cause system damage. Therefore, it is of great practical significance to monitor the aging of IGBT module bond wires.

Since the aging degree of the bond wires encapsulated in the IGBT module cannot be directly observed, it is necessary to evaluate the aging state of the bond wires by other electrical parameters, such as the overshoot of the turn-on gate voltage [5], $V_{c e}$ [6], on-state resistance [7], turn-on voltage drop [8]. Studies have shown that electromagnetic interference (EMI) signals can also be used as electrical parameters for aging monitoring [9]. In [10], the IGBT chip is simulated as a magnetic dipole, and radiated interference signal is emitted into space. When the radiation interference signal is captured by the near-field coil, the different operation states of the chip can be judged according to the difference of the interference signal. In [11], the EMI sources inside the three-phase inverter are simulated as voltage source and current source respectively, and the common mode $(\mathrm{CM})$ interference and DM interference in the circuit are predicted. In the traditional model, the DM interference and CM interference are independent of each other, but the DM interference affects the CM interference, and the $\mathrm{CM}$ interference does not affect the DM interference. Therefore, it is not necessary to consider the influence of CM interference signal when aging monitoring by DM interference signal. In [12], the peak value of DM interference signal generated in the turn-off process of IGBT module is used to monitor the aging degree of bond wires, which can not only avoid considering the influence of junction temperature of IGBT module, but also directly monitor the aging degree of bond wires under working conditions without damaging the packaging of IGBT module. However, in practical application, the amplitude measurement of DM interference signal usually requires expensive

mail:2435189825@qq.com,_27473538@qq.com,_dumx@tjut.edu.cn, zo@elektro.dtu.dk)

Ziwei Ouyang is also with Department of Electrical Engineering, Technical University of Denmark, Lyngby, Denmark.

Jing Hu is with School of Energy and Safety Engineering, Tianjin Chengjian University, Tianjin, China. (e-mail: hujingemail@163.com) 
equipment such as spectrum analyzer, which improves the cost and reduces the practicability of the method. In conclusion, based on the study of the aging mechanism of bond wires inside IGBT module and the PFCs of DM interference signal, it is found that the PFCs of DM interference signal is directly related to the aging state of IGBT module bond wires. Based on this, this paper proposes an on-line monitoring method of bond wires aging based on PFCs of DM interference signal generated by IGBT module switching process.

The rest of this paper is organized as follows. In the second part, the PFCs of the DM interference signal after the bond wires lift-off of IGBT module are studied. When the collector current rise time in the turn-on process is the same as the collector current fall time in the turn-off process, the collector current in the switch process is equivalent to isosceles trapezoid. On this basis, the frequency domain analysis is carried out, and the phase relationship between the bond wires aging degree and the DM interference signal is obtained. In the third part, the method of obtaining the phase of DM interference signal is proposed. In the fourth part, the change rule of the phase of the DM interference signal under different aging degree of the bond wires of IGBT module is verified by experiments, and the conclusion that the phase of the DM interference signal decreases with the increase of the number of the lift-off bond wires is obtained. The fifth part summarizes the whole paper.

\section{Phase ANALYsis OF DM INTERFERENCE SIGNAL AFTER BOND WIRES AGING}

The schematic diagram of Buck converter in the continuous mode is shown in Fig. 1 [12] [13]. The $d i / d t$ caused by the fastswitching behavior of IGBT chip is the main source of EMI in the system. The high $d i / d t$ is coupled with the parasitic parameters of IGBT module itself and the line parameters to produce DM interference.

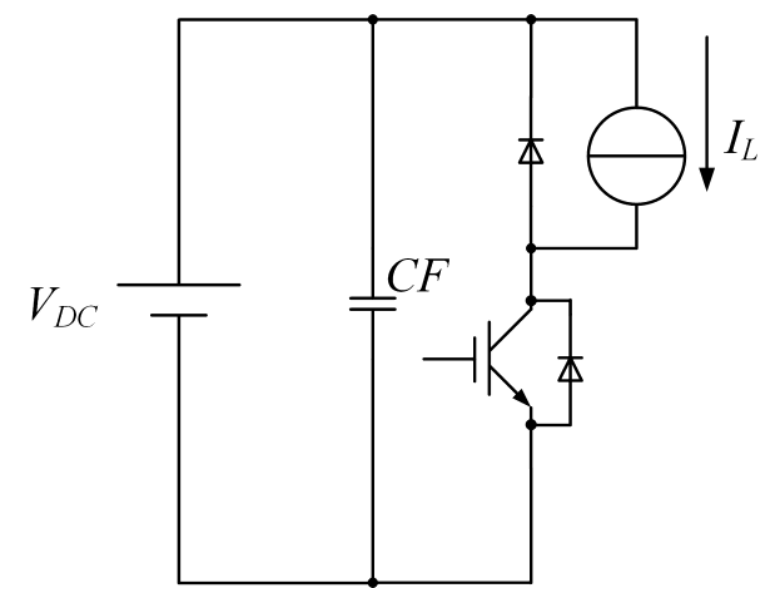

Fig. 1. Schematic diagram of Buck Converter

\section{A. Switching behavior of IGBT chip}

The waveforms of gate voltage and collector current in the switching process of IGBT chip are shown in Fig. 2.

In Fig. 2, $I_{\text {peak }}$ is the current spike caused by diode reverse recovery current, and $I_{0}$ is the load current in steady state. From time $t_{1}$ to $t_{2}$, when the gate voltage gradually increases from the

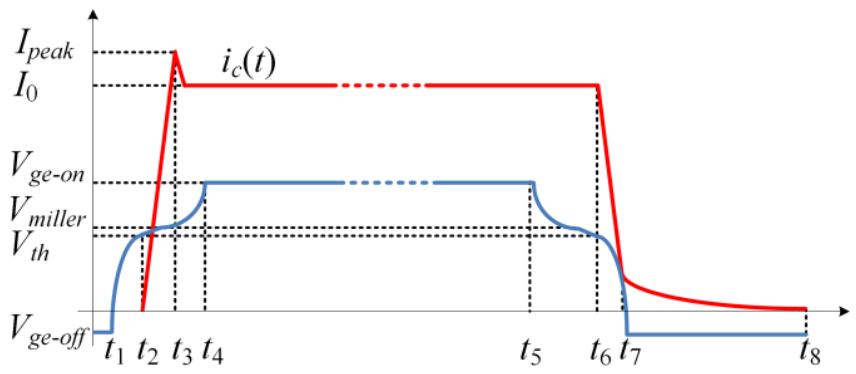

Fig. 2. Waveform of gate voltage and collector current during IGBT chip switching

off voltage $V_{g e-o f f}$ to the threshold voltage $V_{t h}$, the IGBT chip starts to turn on, and the collector current $I_{c}$ increases rapidly. At time $t_{3}$, due to the reverse recovery current of the diode, $I_{c}$ exceeds the load current $I_{0}$ in the stable state, forming a current peak $I_{\text {peak }}$. During the turn off process, after time $t_{6}$, the gate voltage decreases below the threshold voltage $V_{t h}$, the IGBT chip starts to turn off, and the collector current decreases rapidly until time $t_{7}$, when the tail current begins to form. During the switching process, the current change rate of the collector depends on the carrier concentration in its internal channel, and the rapid change rate of collect current is shown in formula (1) [11].

$$
\frac{d I_{c}}{d t}=\sqrt{\frac{2 I_{c}}{\left(1-\alpha_{p п D}\right)} \mu C_{o x} \frac{W}{L}} \frac{d V_{g e}}{d t}
$$

The transient model of IGBT chip can accurately describe the current change rate in the switching process, but the calculation of this model is more complex. In order to simplify the DM interference analysis, it is assumed that the collector current rise time $t_{r 1}=t_{3}-t_{2}$ and fall time $t_{r 2}=t_{7}-t_{6}$ are the same in the switching process of IGBT chip, and then it is regarded as an ideal isosceles trapezoid function, as shown in Fig. 3.

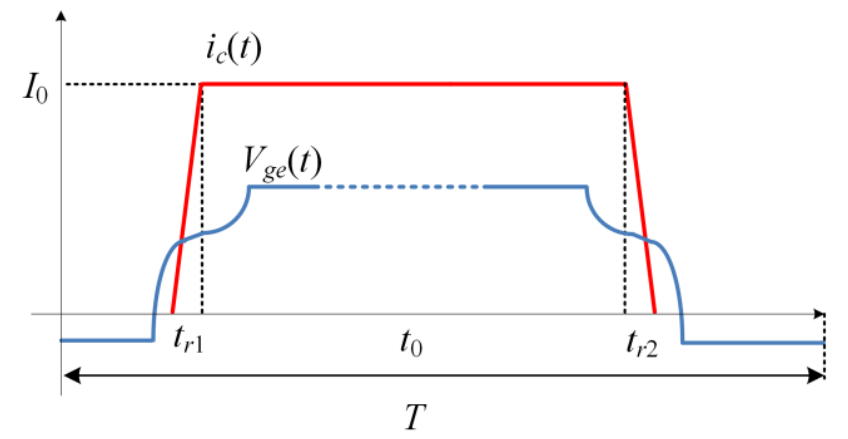

Fig. 3. Approximate equivalent waveform of collector current in IGBT chip switching process

In Fig. 3, when $t_{r 1}=t_{r 2}=t_{r}$, the DM interference sources in the switching process are approximately the same. On this basis, the DM interference signal generated by IGBT chip switch is analyzed. Under the assumption, the collector current in the switching process of IGBT chip is approximately isosceles trapezoid, and the FFT expansion of the isosceles trapezoid interference source is shown in formula (2) [14]. 


$$
I(t)=I_{0} \tau / T+\sum_{n=1}^{\infty} C_{n} \cos \left(n \omega t+\varphi_{n}\right)
$$

Where $\tau=t_{0}+t_{r} ; C_{n}=2 I_{0}\left[\left(t_{0}+t_{r}\right) / T\right]\left\{\sin \left[n \pi\left(t_{0}+t_{r}\right) / T\right] /\left[n \pi\left(t_{0}+\right.\right.\right.$ $\left.\left.\left.t_{r}\right) / T\right]\right\}\left\{\left[\sin \left(n \pi t_{r}\right) / T\right] /\left[\left(n \pi t_{r}\right) / T\right]\right\} ; \varphi_{n}=-n \pi\left(\tau+t_{0}\right) / T$.

The behavior model of IGBT chip is shown in Fig. 4.

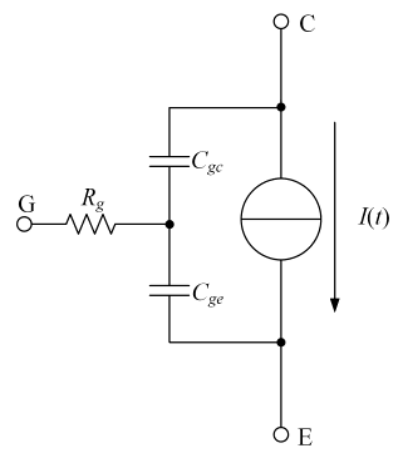

Fig. 4. Behavior model of IGBT chip

In Fig. $4, C_{g c}$ is the parasitic capacitance between the collector and the gate of the chip, $C_{g e}$ is the parasitic capacitance between the gate and the emitter, and the parasitic capacitance is determined by the length and width of the channel inside the chip; $R_{g}$ is the gate parasitic resistance.

\section{B. Aging analysis}

The collector terminal, IGBT chip, emitter terminal and diode chip in IGBT module are connected to copper layer on DBC by bond wires. Bond wires lift-off is one of the common aging forms. The common bond wires lift-off is that the bond wires between emitter and copper layer on DBC has different shear stress due to different CTE. The model diagram and equivalent circuit diagram before and after the bond wires inside the module lift off are shown in Fig. 5(a) and (b) respectively[12][15].

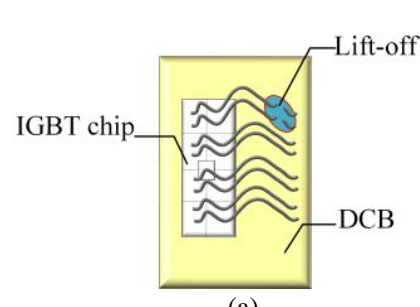

(a)

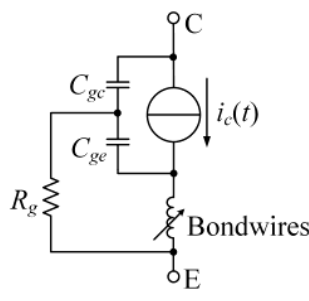

(b)
Fig. 5. (a) IGBT module model diagram (b) Equivalent circuit of IGBT module

In Fig. 5 (a), the eight parallel bond wires can actually be regarded as a variable equivalent inductance in the switching process of IGBT module. The inductor describes a part of the current loop and is the same loop for all wires. The parallel inductance in the equivalent circuit shows that the inductance between the chip and the module emitter terminal increases when the bond wires lift off. Compared with the healthy IGBT module, the equivalent inductance of the bond wires is larger and the ability to hinder the current change is stronger, which causes the current phase angle to change.

According to the application condition of the Norton's equivalent theorem, the one port network composed of linear power supply and impedance can be equivalent to the parallel connection of current source and equivalent impedance. Therefore, IGBT module is equivalent to the parallel connection of internal parasitic parameters and bond wires impedance. The Norton's equivalent circuit diagram is shown in Fig. 6.

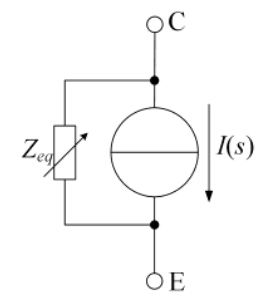

Fig. 6. Norton equivalent circuit of IGBT module

Where $Z_{e q}$ is the equivalent impedance of the double terminal network after the current source is set to zero, as shown in formula (3).

$$
Z_{e q}=\frac{1}{j \omega C_{g c}}+\frac{R_{g}\left(\frac{1}{j \omega C_{g e}}+j \omega L_{\text {bondwires }}\right)}{R_{g}+\frac{1}{j \omega C_{g e}}+j \omega L_{\text {bondwires }}}
$$

In order to determine the influence of the bond wires lift off on the impedance angle of the equivalent impedance, the formula (3) is transformed into algebraic form and polar coordinate form, as shown in formulas (4) and (5), respectively.

$$
\begin{gathered}
Z_{e q}=\frac{R_{g}\left(\frac{1}{\omega C_{g e}}-\omega L_{\text {bondwires }}\right)^{2}-j R_{g}{ }^{2}\left(\frac{1}{\omega C_{g e}}-\omega L_{\text {bondwires }}\right)}{R_{g}{ }^{2}+\left(\frac{1}{\omega C_{g e}}-\omega L_{\text {bondwires }}\right)^{2}}-j \frac{1}{\omega C_{g c}} \\
Z_{e q}=\frac{R_{g}\left(\frac{1}{\omega C_{g e}}-\omega L_{\text {bondwires }}\right)}{\sqrt{R_{g}{ }^{2}+\left(\frac{1}{\omega C_{g e}}-\omega L_{\text {bondwires }}\right)^{2}} \angle \theta+\frac{1}{\omega C_{g c}} \angle-90^{\circ}}
\end{gathered}
$$

As shown in formula (4), when the frequency is low, the imaginary part of the first term is negative, and the overall equivalent impedance is capacitive, so the phase angle corresponding to the first term in formula (5) is negative, it is shown in formula (6).

$$
\angle \theta=-\arctan \frac{R_{g}}{\left(\frac{1}{\omega C_{g e}}-\omega L_{\text {bondwires }}\right)}
$$

It can be seen from formula (5) and (6) that the impedance angle decreases with the increase of the number of bond wires lift off, which leads to the increase of the equivalent impedance $Z_{e q}$ amplitude and the decrease of the impedance angle.

\section{DM interference modeling of actual working conditions}

The equivalent circuit of DM interference in Buck Converter shown in Fig. 1 is shown in Fig. 7. 


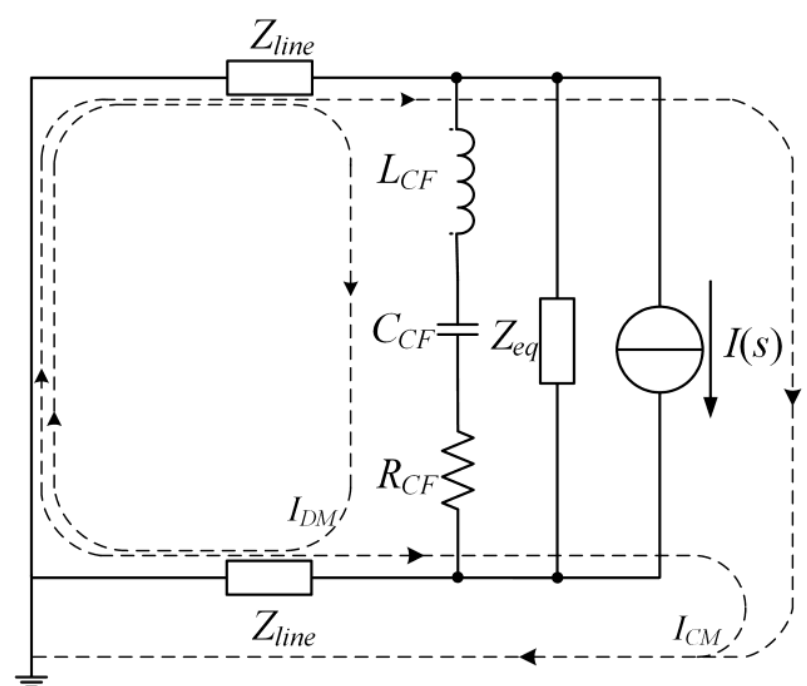

Fig. $\overline{\overline{7}}$. Conducted interference equivalent circuit of buck converter under practical conditions

According to the circuit theory, the equivalent circuit of DM interference is analyzed and shown in formula (7).

$$
I_{D M}=\frac{Z_{C F} Z_{e q}}{\left(2 Z_{\text {line }} Z_{C F}+Z_{C F} Z_{e q}+2 Z_{\text {line }} Z_{e q}\right)} I(s)
$$

In formula (7), $Z_{C F}$ is the frequency domain equivalent impedance of the capacitor $\mathrm{CF}, Z_{\text {line }}$ is the equivalent impedance of the connecting wire, and $I_{D M}$ is the DM current flowing through the connecting wire. In order to show the effect of bond wires lift-off on the phase of DM interference current more clearly, formula (7) is further simplified, as shown in formula (8) and (9).

$$
\begin{gathered}
I_{D M}=\frac{Z_{4}}{\left(2 \frac{Z_{3} Z_{4}}{Z_{e q}}+Z_{4}+2 Z_{3}\right)} I(s) \\
I_{D M}=\left|I_{D M}\right| \angle \alpha
\end{gathered}
$$

\section{ON LINE MONITORING SYSTEM FOR BOND WIRES AGING}

The process of on-line monitoring the aging state of internal bond wires of IGBT module through the phase angle characteristics of DM interference signal is shown in Fig 8.

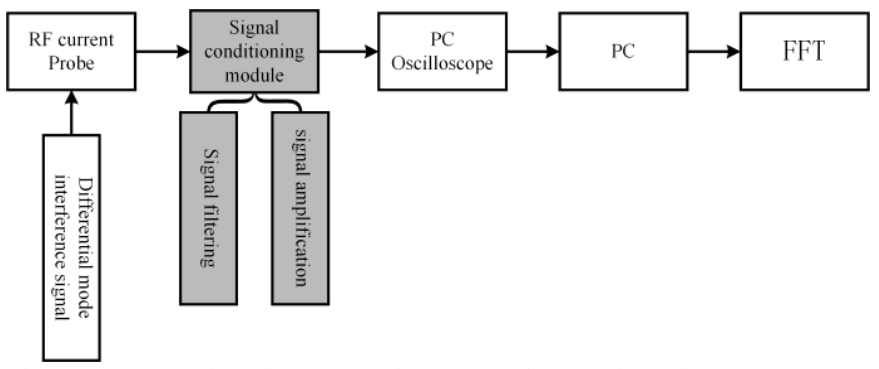

Fig. 8. Process of on-line monitoring system for bonding wire aging

As can be seen from fig. 8, firstly, the DM interference signal is sensed and extracted by the RF current probe; Secondly, the DM interference signal is filtered by low-pass filter to reduce the difficulty of signal acquisition; Thirdly, since the amplitude of the interference signal is small and the accuracy of the judgment index is higher, the oscilloscope cannot directly identify and effectively collect, so it must be amplified through the signal amplifier, so that it can be easily identified and collected by the oscilloscope and transmitted to the computer; Finally, the PFCs of DM interference signal are obtained by Fast Fourier Transform (FFT) of time domain signal in computer.

\section{A. low pass filter}

In the previous study, the amplitude of the DM interference current in the frequency range of $150 \mathrm{kHz} \sim 2 \mathrm{MHz}$ is directly related to the number of lift-off bond wires, and there is no regular relationship between the amplitude of the DM current and the number of lift-off bond wires in the frequency range above 2MHz, as shown in Fig. 9.

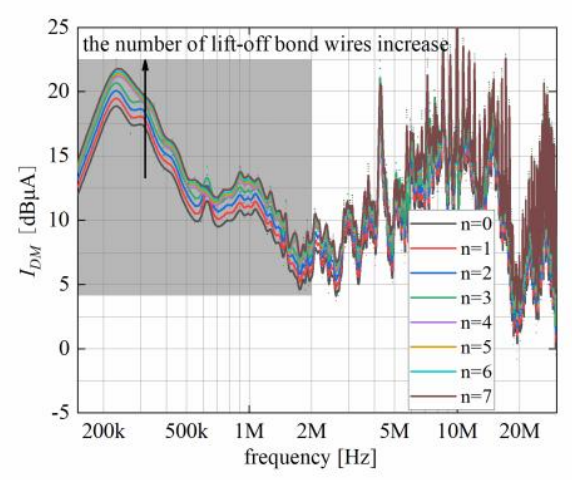

Fig. 9. The relationship between the number of lift-off bond wires and EMI current

It can be seen from Fig. 9 that the DM interference signal has no relationship with the number of lift-off bond wires in the frequency range above $2 \mathrm{MHz}$. Only the PFCs of DM EMI signal in low frequency band are directly related to the number

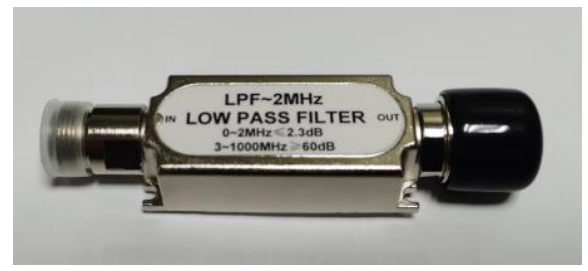

(a)

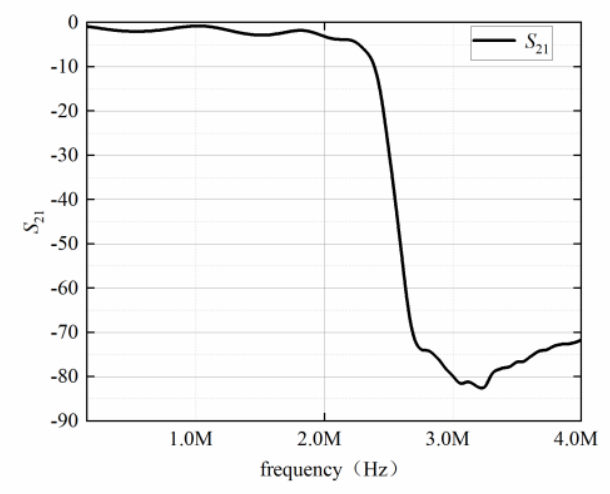

(b)

Fig. 10. (a) physical diagram (b) insertion loss of low pass filter 
of lift-off bond wires in IGBT module. Based on this, a $2 \mathrm{MHz}$ low-pass filter is used to filter the interference signal in higher frequency band, reduce the interference introduced by the signal amplification part, and improve the accuracy and reliability of monitoring results. In order to avoid the influence of the access filter on the accuracy of the measured signal, the insertion loss $S_{21}$ of the filter is measured by the Vector Network Analyzer. The physical picture of the filter and its insertion loss $S_{21}$ are shown in Fig. 10 (a) and (b) respectively.

It can be seen from Fig. 10 that the insertion loss is close to zero below $2 \mathrm{MHz}$, which indicates that $\mathrm{DM}$ interference signal below $2 \mathrm{MHz}$ has almost no loss when passing through the lowpass filter, so the access to the low-pass filter does not change the load power of the frequency band below $2 \mathrm{MHz}$, and it does not affect the measurement results. The insertion loss is -80 to $-70 \mathrm{~dB}$ above $3 \mathrm{MHz}$, which indicates that the DM interference signal above $3 \mathrm{MHz}$ is almost completely filtered after passing through the low-pass filter to avoid the influence of this frequency band on the measurement results after the amplifier is connected.

\section{B. signal amplifier}

After the DM interference signal of the required frequency band is output by the filter, because its signal amplitude is small and its power is not enough to be measured by the data acquisition module, it needs to be amplified. In the whole signal amplifier, there are three parts: input matching circuit, output matching circuit and bias circuit. The physical diagram and schematic diagram of the designed low noise $1 \mathrm{kHz}-2 \mathrm{GHz}$ @ $32 \mathrm{~dB}$ RF small signal high gain amplifier are shown in Fig. 11 (a) and (b) respectively.

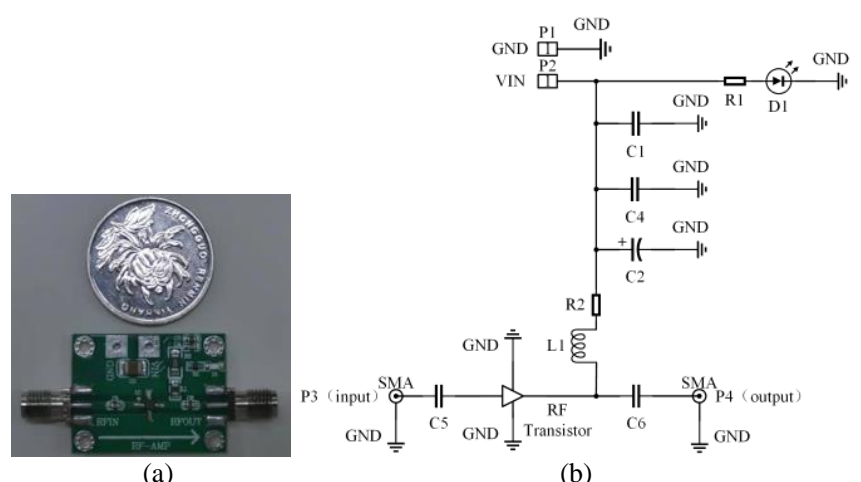

(a)

(b)

Fig. 11. Low noise small signal high gain RF amplifier (a) physical diagram (b) schematic diagram

In Fig. 11(b), $C_{1}, C_{2}$ and $C_{4}$ are power filter capacitors; $C_{5}$ and $C_{6}$ are the proper size of DC isolation capacitors. In the amplifier, the size of DC isolation capacitor affects the cut-off frequency of the working frequency band. Due to the skin effect, the capacitor will show certain high frequency characteristics at high frequency, which leads to the capacitor is not only a simple capacitor but also a high pass filter. The smaller the capacitance, the higher the cut-off frequency and the higher the high frequency loss; On the contrary, the larger the capacitance is, the lower the cut-off frequency and the lower the high frequency loss. In this design, $100 \mathrm{pF}$ is selected. $R_{2}, L_{1}$ and $C_{6}$ constitute a DC bias circuit. The larger the inductance of the bias part is, the lower the cut-off frequency is. However, the high-frequency characteristic is poor, which is prone to harmonics. The smaller the inductance is, the higher the cut-off frequency is, and the better the high-frequency characteristic is, so inductance is selected $8.2 \mu \mathrm{H}$. In order to test the performance of the amplifier, its gain characteristics are measured by oscilloscope, as shown in Fig. 12.

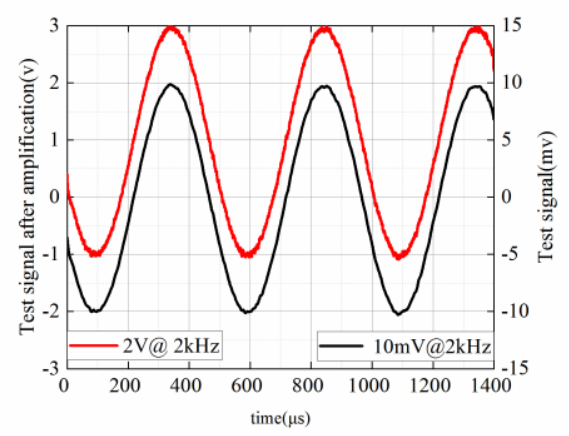

Fig. 12. Gain of signal amplifier

It can be seen from Fig. 12 that when the input sine wave signal of the amplifier is $V_{P P}=20 \mathrm{mV}$ and $f=2 \mathrm{KHz}$, the output signal of the amplifier is $V_{P P}=4 \mathrm{~V}$ and $f=2 \mathrm{KHz}$. According to the comparison before and after the input of the amplifier signal, the voltage magnification of the amplifier is 200 when the power supply of the amplifier is $9.8 \mathrm{v}$. When the difference of current signal in frequency domain is $0.2 \mathrm{~dB} \mu \mathrm{A}$, the difference is $0.205 \mathrm{~mA}$ after amplifier amplification, which make ensure the DM signal can be recognized by the oscilloscope.

\section{DM interference signal monitoring system}

After signal conditioning circuit processing such as low pass filter and small signal high gain amplifier, the required DM interference signal can be obtained. The measurement system is shown in Fig. 13.

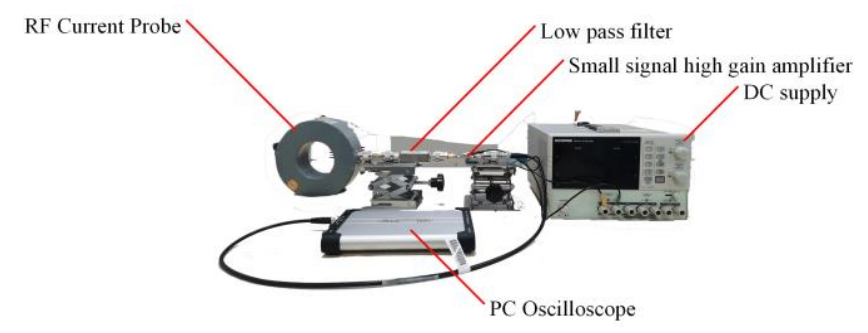

Fig. 13. Implementation of DM interference signal monitoring system

As can be seen from Fig. 13, the RF current probe senses the DM interference signal, selects the DM interference signal below $2 \mathrm{MHz}$ frequency band through the low-pass filter, and amplifies the DM interference signal 200 times through the small signal high gain amplifier to ensure that the PC oscilloscope board can accurately identify, and the DC power supply provides the working voltage for the small signal amplifier. 


\section{EXPERIMENTAL VERIFICATION}

\section{A. Experimental platform}

The DM interference test platform under actual conditions is shown in Fig. 14.

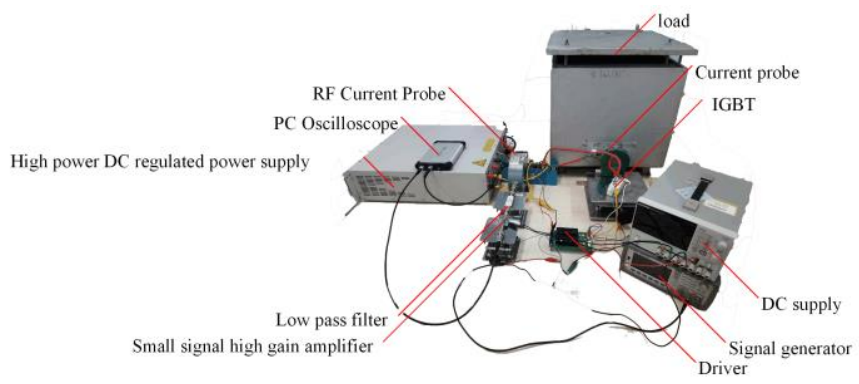

Fig. 14. DM interference test experimental platform

It can be seen from Fig. 14 that the DM interference current signal under the actual working condition is obtained by coupling the current probe, processed by the low-pass filter and signal amplifier, and then input to the PC through the oscilloscope board card; The time domain DM current signal measured by oscilloscope board is transformed into frequency phase angle curve by FFT in PC.

The switching frequency of IGBT module is set to $1 \mathrm{kHz}$ and the duty cycle is set to $50 \%$. The aging degree of IGBT module bond wires was changed, and the frequency phase relationship curve of DM interference signal was mapped. According to the measurement results, the PFCs of DM interference signal in buck converter circuit with healthy IGBT module and aging IGBT module are compared, and the aging state of bond wires of IGBT module is identified.

\section{B. Effect of bond wires aging on PFCs of DM interference signal}

In order to verify the influence of aging degree of bond wires on the PFCs of DM interference in Buck converter, different aging degrees can be simulated by cutting off the bond wires in IGBT module step by step, and the PFCs of DM interference signal in Buck converter can be measured. When the bond wires of IGBT module is in different aging degrees, the difference of PFCs of DM interference current of Buck Converter measured

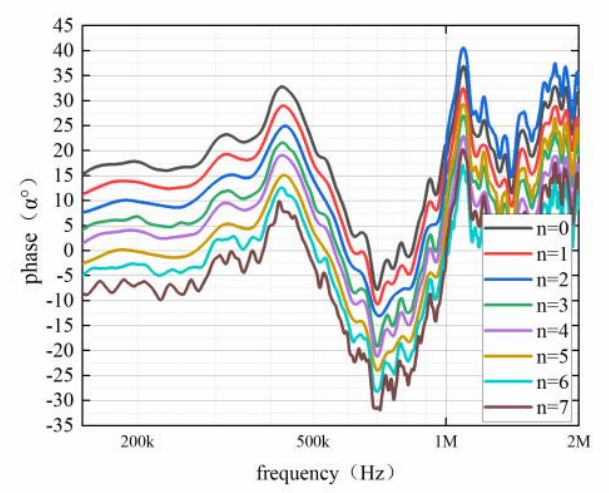

Fig. 15. Phase frequency relation curve between the number of lift-off bond wires and DM interference signal is shown in Fig. 15.

In Fis. $15, n$ represents the number of lift-off bond wires. The relationship between the number of lift-off bond wires and the phase of interference signal is shown in the figure. Compared with the higher frequency section, the influence of the bond wires lift-off on the PFCs of the lower frequency segment DM interference is more obvious. The phase angle of DM interference signal increases with the increase of frequency in the range of $150 \mathrm{kHz}$ to $450 \mathrm{kHZ}$; The phase angle of DM interference signal decreases with the increase of frequency in the range of $450 \mathrm{kHZ}$ to $700 \mathrm{kHz}$. In the range of $150 \mathrm{kHz}$ to $1 \mathrm{MHz}$, the phase of DM interference signal decreases with the increase of the number of lift-off wires. In the frequency range of more than $1 \mathrm{MHz}$, the relationship between the number of the bond wires lift-off and the phase of DM interference is not obvious, which is because the influence of parasitic parameters and distribution parameters in high frequency cannot be ignored and the influence of external noise interference cannot be eliminated in the actual working conditions. Therefore, when using high frequency DM interference voltage and DM interference current signal to monitor the aging of IGBT bond wires, the bond wires cannot be equivalent to variable inductance only, but also the influence of the equivalent resistance and parasitic capacitance of bond wires on the circuit model is also needed.

\section{Aging monitoring of bond wires}

The aging of bond wires in IGBT module is a gradual process, so it is necessary to continuously obtain the PFCs of current EMI signal and monitor the aging degree of bond wires. In the range of $20 \mathrm{kHz}$ to $1 \mathrm{MHz}$, the phase angle characteristic of DM interference signal generated by IGBT module is obviously related to the aging degree of bond wires.

Under the actual working condition, the RF current probe is used to measure the DM interference signal generated by IGBT module under different aging degrees of bond wires, and the relationship between DM interference phase angle characteristics and the number of lift-off bond wires is given. The measurement results are shown in Table I.

TABLE I

DM INTERFERENCE PHASE ANGLE PEAK VALUE OF IGBT MODULE AFTER LOW FREQUENCY BONDING WIRE LIFT OFF

\begin{tabular}{cc}
\hline \hline $\begin{array}{c}\text { The Number of } \\
\text { lift-off bond wires }\end{array}$ & Phase $\left(\alpha^{\circ}\right)$ \\
\hline 0 & $32.79^{\circ}$ \\
\hline 1 & $28.98^{\circ}$ \\
\hline 2 & $24.95^{\circ}$ \\
\hline 3 & $21.61^{\circ}$ \\
\hline 4 & $19.08^{\circ}$ \\
\hline 5 & $15.11^{\circ}$ \\
\hline 6 & $12.56^{\circ}$ \\
\hline 7 & $9.77^{\circ}$ \\
\hline \hline
\end{tabular}

It can be seen from Table I that the phase angle of DM interference signal decreases with the increase of the number of 
lift-off bond wires, and the decreasing trend is basically unchanged. The DM interference change value of each module is not the same, only from the phase difference is not enough to accurately represent the aging degree, so the aging monitoring index parameter $\mathrm{K}$ is defined as $\mathrm{K}=\left(\alpha_{0}-\alpha\right) / \alpha_{0}$, where $\alpha_{0}$ is the maximum phase angle of DM interference signal in the range of $150 \mathrm{kHz} \sim 700 \mathrm{kHz}$ when IGBT module is healthy; $\alpha$ is the maximum phase angle of DM interference signal in the current state. And the relationship curve between $\mathrm{K}$ and the number of lift-off bond wires is shown in Fig. 16.

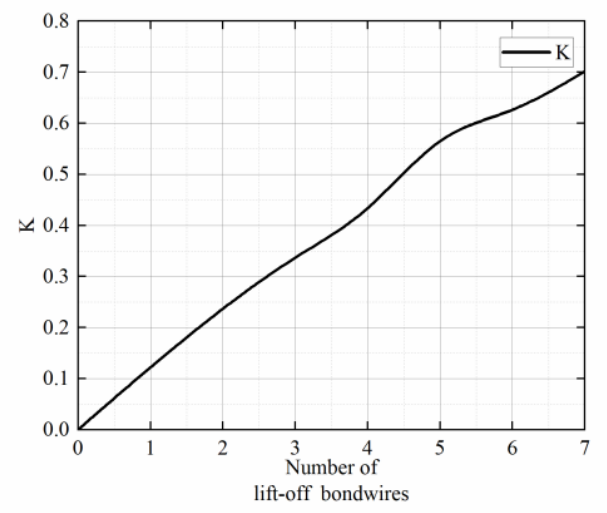

Fig. 16. The relationship curve between aging index $\mathrm{K}$ and the number of bond wires lift off

It can be seen from Fig. 16 that the aging monitoring index parameter $\mathrm{K}$ increases with the increase of the number of lift off bond wires, and the linearity between $\mathrm{K}$ value and the number of lift-off bond wires is prominent. In general, the relationship between the number of lift-off bond wires and on-state resistance is used to monitor the aging state of bond wires. The relationship is shown in Fig. 17.

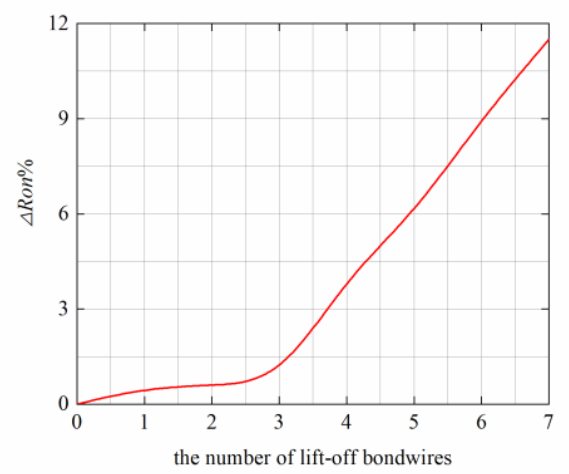

Fig. 17. The relationship curve between the number of bond wires lift-off and the percentage of on state resistance

As can be seen from Fig.17, when the aging degree of the bond wires is light, the change of the impedance is very small. With the deepening of the aging degree, the on-state resistance changes nearly exponentially. When one bond wire is lift-off, the change of on resistance is less than $1 \%$, which is very small compared with the healthy IGBT module. The change of onstate resistance of two bond wires lift off is almost the same as that of one. When three bond wires are lift off, the on resistance changes obviously. Therefore, when the aging degree is light, the on-state resistance is not sensitive as the electrical parameter of the bond wire aging monitoring, and cannot accurately monitor the aging degree of the IGBT module bond wires. Only after a certain number of bond wires are lift-off, the on-state resistance can effectively identify whether the IGBT module bond wire is aging. It can be seen from Fig.16 and Fig.17 that the PFCs linearity of the DM interference signal is higher than that of the on-state resistance change percentage in monitoring the aging state of the bond wires of IGBT module, and it can be accurately monitored even the aging degree of the bond wire is light. Generally, the IGBT module with four lift-off bond wires needs to be replaced. Comparing the relationship between onstate resistance, PFCs of DM interference signal and bond wires aging, when the obtained bond wire aging monitoring index $\mathrm{K}$ reaches about 0.4 , it can be considered that more than 4 bond wires lift off and IGBT module needs to be replaced.

\section{CONCLUSION}

In this paper, a method based on the phase angle characteristic of DM interference signal is proposed to monitor the bond wires condition of IGBT module, and the relationship between the phase of DM interference signal and the aging degree of bond wires is established. According to the theoretical and experimental results, the phase angle of DM interference signal decreases with the aging degree of IGBT bond wires. According to the aging degree of bond wires and PFCs of DM interference signal, when the calculated aging parameter $\mathrm{K}$ reaches 0.4 , it is considered that more than four bond wires lift off, and the aging device should be replaced in time to avoid the impact of aging on the reliability of power converter. Because the measurement method proposed in this paper can realize the real-time phase angle measurement of DM interference current signal, and then can accurately monitor the aging state of IGBT module bond wires. Beside, the research method in this paper provides an approximate linear monitoring method for bonding wire lift-off. In practical application, only one set of RF current probe, appropriate low-pass filter, signal amplifier and oscilloscope board are needed to measure the PFCs of DM interference signal, and realize the aging monitoring of IGBT module bond wires.

\section{REFERENCES}

[1] S. Yang, A. Bryant, P. Mawby, D. Xiang, L. Ran, and P. Tavner, "An industry-based survey of reliability in power electronic converters," IEEE Trans. Ind. Appl., vol. 47, no. 3, pp. 1441-1451, May/Jun. 2011.

[2] V. Smet et al., "Ageing and failure modes of IGBT modules in high temperature power cycling," IEEE Trans. Ind. Electron., vol. 58, no. 10, pp. 4931-4941, Oct. 2011.

[3] M. Ciappa, "Selected failure mechanisms of modern power modules," Microelectronics Rel., vol. 42, nos. 4-5, pp. 653-667, 2002.

[4] X. Wang, P. Sun, L. Sun, Q. Luo and X. Du, "Online Condition Monitoring for Bond Wire Degradation of IGBT Modules in Three-Level NeutralPoint-Clamped Converters," IEEE Trans. Ind. Electro, vol. 68, no. 8, pp. 7474-7484, Aug. 2021, doi: 10.1109/TIE.2020.3005073.

[5] X. Wang, P. Sun, L. Sun, Q. Luo and X. Du, "Online Condition Monitoring for Bond Wire Degradation of IGBT Modules in Three-Level NeutralPoint-Clamped Converters," IEEE Trans. Ind. Electro, vol. 68, no. 8, pp. 
7474-7484, Aug. 2021, doi: 10.1109/TIE.2020.3005073.

[6] A. Singh, A. Anurag and S. Anand, "Evaluation of Vce at Inflection Point for Monitoring Bond Wire Degradation in Discrete Packaged IGBTs," in IEEE Trans. Power Electro, vol. 32, no. 4, pp. 2481-2484, April 2017, doi: 10.1109/TPEL.2016.2621757.

[7] Guang Zeng, Haiyang Cao, Weinan Chen, Josef Lutz, "Difference in DeviceTemperature Determination Using p-n-Junction Forward Voltage and Gate Threshold Voltage , "IEEE Trans. Power Electron, vol.34, no.3, pp2781-2793, March 2019

[8] Jie Chen, Erping Deng, Luhong Xie, "Investigations on Averaging Mechanisms of Virtual Junction Temperature Determined by VCE(T) Method for IGBTs," IEEE Trans. Electron Devices, vol.67, no.3, pp11061112, March2020

[9] J. An, C. Zhuang, F. Rachidi and R. Zeng, "An Effective EMTR-Based High-Impedance Fault Location Method for Transmission Lines," IEEE Trans. Electro. Compa, vol. 63, no. 1, pp. 268-276, Feb. 2021, doi: 10.1109/TEMC.2020.2991862.

[10] Rajashree Biswas, Aurobinda Routray, Shuvam Chakraborty, "EMR Signature Analysis for Health monitoring and early stage fault diagnosis of IGBT,'IECON 2017 - 43rd Annual Conference of the IEEE Industrial ElectronicsSociety

[11]Wu Zhou, Xuejun Pei, “A New EMI Modeling Method for Mixed-Mode

Noise Analysis in Three-Phase Inverter System," IEEE Access , vol.8, no. pp71535-71547, March 2020

[12]C. Chu, C. Dong, M. Du, X. Zhou and Z. Ouyang, "Aging Monitoring of Bond Wires Based on Differential Mode Conducted Interference Spectrum for IGBT Module," IEEE Trans. Elect. Compa, doi: 10.1109/TEMC.2021.3052904

[13] Jin Meng, Weiming Ma, Qijun Pan, Lei Zhang, Zhihua Zhao, "Multiple Slope Switching Waveform Approximation to Improve Conducted EMI Spectral Analysis of Power Converters," IEEE Trans. Elect. Compa., vol. 48, no. 4, pp 724-751, Nov. 2006, DOI: 10.1109/TEMC.2006.882859.

[14]F. Costa and D. Magnon, "Graphical analysis of the spectra of EMI sources in power electronics," IEEE Trans. Power Electro, vol. 20, no. 6, pp. 14911498, Nov. 2005, doi: 10.1109/TPEL.2005.857564.

[15] C. Chen et al., "Signal Sweeping Technique to Decouple the Influence of Junction Temperature and Bondwire Lift-off in Condition Monitoring for Multichip IGBT Modules," CIPS 2018; 10th International Conference on Integrated Power Electronics Systems, Stuttgart, Germany, 2018, pp. 1-6.

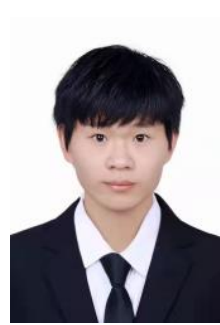

Chengpeng Chu received the B.S. degree in electrical engineering in 2019 from the Tianjin University of Technology, Tianjin, China, where he is currently working toward the M.S. degree in control science and engineering with the College of Electrical and Electronic Engineering.

His major research interests include condition monitoring for power electronics system and electromagnetic compatibility technology.

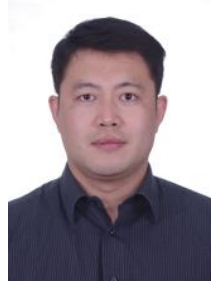

Chao Dong received the B.S. degree in automation engineering from the Tianjin University of Technology, Tianjin, China, in 2000 and the M.S. degree in computer engineering from Tianjin University, Tianjin, China, in 2006. Since 2000, has been working with the Tianjin University of Technology as an Associate Professor. His research interests include power electronics system reliability, electromagnetic compatibility technology, process industry control technology, etc.

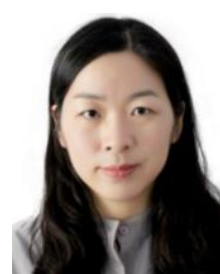

Jing Hu received her Ph.D. degree from Tianjin University (TJU), Tianjin, China, in 2014. Since 2005, she has also been an associate professor with the School of Energy and Safety Engineering, Tianjin Chengjian University, Tianjin.

Her main research interests are heat and mass transfer technology.

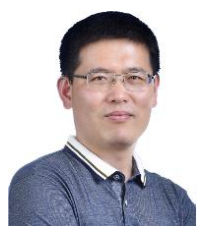

Mingxing Du received the Ph.D. degree from Tianjin University (TJU), Tianjin, China, in 2012. Since 2006, he has also been an associate professor with the School of Electrical and Electronic Engineering, Tianjin University of Technology, Tianjin. He was a visiting scholar with the Power Electronics Research Centre in the National University of Ireland (Galway) in 2018.

His main research interests are condition monitoring for power electronics, power semiconductor module thermal modeling, and electromagnetic compatibility in power electronics system.

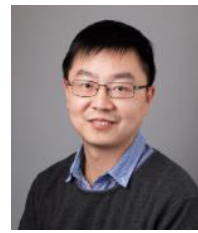

Ziwei Ouyang(S'07, M'11, SM'17) received his $\mathrm{PhD}$ degree from Technical University of Denmark (DTU) in 2011. From 2011 to 2013, he was a postdoc researcher at DTU. From 2013 to 2016, he was appointed as an assistant professor at the same department. Since from April 2016, he is an associate professor at DTU. His research areas focus on high-frequency planar magnetics modeling and integration, high-density high-efficiency power converters, PV battery energy storage system, and wireless charging etc.

$\mathrm{He}$ is IEEE senior member. He has over 60 high impact IEEE journal and conference publications, co-author on a book chapter on Magnetics for the "Handbook of Power Electronics" and currently he is the holder of 8 international patents. He was a recipient of Young Engineer Award at PCIMAsia 2014, and received Best Ph.D. Dissertation of the Year Award 2012 in Technical University of Denmark. He received several Best Paper Awards in IEEE sponsored international conferences. He has been invited to give lectures in many universities, enterprises and educational seminars and workshops around the world including USA, Europe and China. He has served as session chair in some IEEE sponsored conferences and associated editor for IEEE Journal of Emerging and Selected Topics in Power Electronics. 